\title{
Redox Polyelectrolyte Modified Gold Nanoparticles Enhance the Detection of Adenosine in an Electrochemical Split-Aptamer
} Assay

\author{
Lucy L. Coria-Oriundo, ${ }^{[a, b]}$ Helena Ceretti, ${ }^{[c]}$ Yoann Roupioz, ${ }^{[d]}$ Fernando Battaglini ${ }^{*}[a]$
}

[a] Lucy L. Coria-Oriundo, Prof. Fernando Battaglinia, ${ }^{*}$

INQUIMAE (CONICET), Departamento de Química Inorgánica, Analítica y Química Física

Facultad de Ciencias Exactas y Naturales, Universidad de Buenos Aires

Ciudad Universitaria, Pabellón 2, C1428EHA Buenos Aires, Argentina

E-mail: battagli@qi.fcen.uba.ar

[b] Facultad de Ciencias, Universidad Nacional de Ingeniería, Av. Túpac Amaru 210, Lima 25, Perú.

[c] Dr. Helena Ceretti

Universidad Nacional de Gral. Sarmiento, J. M. Gutiérrez 1150, B1613GSX, Los Polvorines, Prov. de Buenos Aires, Argentina.

[d] Dr. Yoann Roupioz

Univ. Grenoble Alpes, CNRS, CEA, SyMMES, 38000 Grenoble, France

Supporting information for this article is given via a link at the end of the document.

\begin{abstract}
We present an electrochemical sandwich-type assay based on the splitting of an aptamer into two fragments. Gold nanoparticles are modified with one of the fragments and a redox polyelectrolyte. The first is used as the recognition element, while the other for the electrochemical signal generation. The splitaptamer used here can detect adenosine, used as a model system for recognizing small molecules. The multiple binding sites on the nanoparticle, along with the high number of redox probes, yield a selective and sensitive assay for adenosine, achieving a limit of detection of $3.1 \mathrm{nM}$ and a linear range up to $75 \mathrm{nM}$. The obtained results are analyzed in terms of the nanoparticle and electrode architectures. The assay can be easily extended to other small molecules and sandwich assays, representing a promising tool for detecting metabolites at the nanomolar level.
\end{abstract}

\section{Introduction}

Metabolites are low molecular-weight molecules, also referred to as small molecules, chemically transformed in biological processes. In clinical chemistry, these molecules represent useful markers for early diagnosis. ${ }^{[1-3]}$ The development of fast and sensitive methods for their detection is an intensive area of research replacing the traditional methods based on chromatographic and spectroscopic techniques, which rely on extensive sample preparation and time-consuming procedures. Sensors represent an alternative, where a selective molecular recognition process occurs, generating a physicochemical change conveniently transduced to an electrical signal. Aptamers have played a key role in the development of sensors since they can be developed for a wide range of targets from metal ions to cells, ${ }^{[4]}$ among them small molecules, where adenosine and cocaine have been extensively used as model systems $^{[5-9]}$. Other features of the aptamers include: its synthesis does not involve animals; during the chemical synthesis, modifications can be introduced at defined positions; these biomolecules are way more stable than proteins and might be dried without loss of activity.

In recent years, the sequence of several aptamers has been split into two parts, maintaining their affinity, ${ }^{[10-16]}$ allowing the detection of a species in a similar way than the antibody sandwich assay. In a sandwich assay using electrochemical signaling, the capture antibody is immobilized on the working electrode surface and binds the analyte. A second labeled antibody subsequently binds to the analyte for the signal generation. Colloidal metal labels have been widely used in the last two decades in this type of assay. ${ }^{[17-19]} \mathrm{A}$ similar approach can be taken with the adenosine aptamer, whose original hairpin sequence can be cut in the loop domain (Scheme 1A, left). [20] The originated segments open a myriad of sandwich-like strategies. These fragments have been used grafted on gold nanoparticles (AuNPs) for colorimetric detection in solution, ${ }^{[7]}$ or on surfaces as recognition elements in biosensors; in the last case, as examples of transduction methods we can mention, fluorescence, ${ }^{[21]}$ surface plasmon resonance (SPR), ${ }^{[6,22]}$ and electrochemical detection. ${ }^{[8,23]}$ Electrochemical detection is a very convenient method since it can be used in turbid samples, a relevant issue in many clinical and biological studies, and due to its hardware simplicity, a low cost per assay can be easily achieved. The use of colloidal metal labels in sandwich assays allows a sensitive response; however, most of the examples presented in the literature involve the nanoparticle's chemical dissolution, involving the addition of reagents and introducing more steps to the detection process or complicated construction schemes. $^{[17,19,23]}$

In this work, we present an electrochemical sandwich assay able to generate an output current at $0.3 \mathrm{~V}$ vs. $\mathrm{Ag} / \mathrm{AgCl}$ using squarewave voltammetry (SWV). The assay is based on the splitting of the adenosine aptamer into two fragments (Scheme $1 \mathrm{~A}$ ), one immobilized on the surface of the electrode (capture fragment, C) and the other one (signaling fragment, S) is immobilized on AuNPs. The AuNPs also contain a redox polyelectrolyte (polyallylamine derivatized with an osmium polypyridyl complex, OsPA) for the signal generation (AuNP/S/OsPA, Scheme 1B). The functionalized nanoparticles play a crucial role in the figures of merit of the assay since each nanoparticle presents several aptamers for adenosine recognition, improving the binding affinity, while the osmium redox centers distributed around all the nanoparticle have a multiplicative effect in the signal generation. In this way, each nanoparticle works as a multidentate ligand improving its binding to the surface, 
achieving state-of-the-art adenosine quantification with a limit of detection (LOD) of $3.1 \mathrm{nM}$.

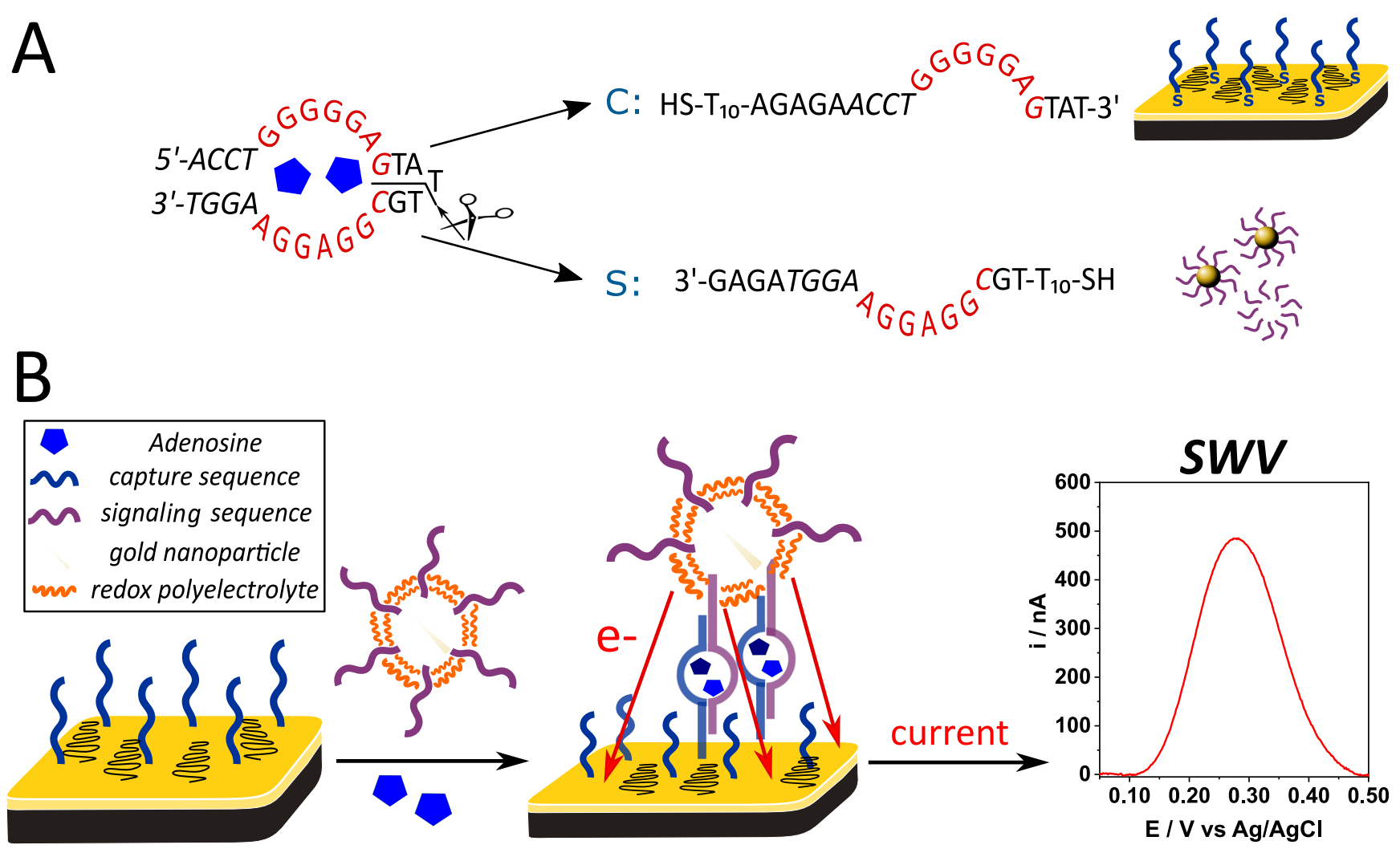

Scheme 1. General principle of the split aptamer-based assays. (A) The original sequence of the adenosine aptamer (left) and the sequences used in this work ( $C$ and $S$, right). Pentagons in blue represent adenosine molecules. (B) Recognition assay: the modified electrode (AuE/C) is exposed to adenosine and AuNP/S/OsPA. After incubation, the amount of adenosine-aptamer complex is determined by square wave voltammetry (SWV).

\section{Results and Discussion}

\subsection{Assay Concept}

Scheme 1A (left) shows the DNA sequence used by Lin and Patel in their structural studies of the DNA-adenosine complex. This single-stranded DNA is composed of 27 nucleotides, which folds into a hairpin structure in the presence of adenosine. The nucleotides forming the loop are rich in guanine and are responsible for the adenosine recognition (depicted as pentagons in the scheme). At the left of the loop, 4 pairs of complementary nucleotides help to stabilize the complex. If this sequence is divided into two fragments by cutting it in the 14th nucleotide (indicated by the scissor in the scheme), it can reassemble in the presence of adenosine.$^{[24]}$ In order to adapt these two new sequences to a sandwich-type assay, some changes were introduced: ${ }^{[7]}$ the top part was selected as capture sequence $(C)$ to be immobilized on a gold electrode surface; a thiol group was added and the sequence extended with thymine nucleotides and a short 5 nucleotide sequence (AGAGA) (Scheme $1 \mathrm{~A}$, right). On the other hand, the bottom part of the sequence $(S)$ was modified at the $5^{\prime}$ terminus with a thiol group followed by 10 thymine nucleotides (Scheme $1 \mathrm{~A}$, right); while in the $3^{\prime}$ end, only four (in italics) of the eight nucleotides match with the complementary $C$ sequence. The $S$ sequence binds to the $C$ sequence only in the presence of adenosine using these modifications. ${ }^{[6,22,25,26]}$

\subsection{Gold nanoparticle functionalization}

Layer by layer assembly is a powerful technique for the construction of complex nanoarchitectures in small areas through a simple method mainly based on electrostatic interactions. ${ }^{[27,28]}$ This technique can be easily applied to charged macromolecules such as polyelectrolytes, ${ }^{[29,30]}$ proteins, [31,32] and DNA. ${ }^{[33]}$ Considering that the aptamer and the redox polyelectrolyte have opposite charges, we decided to combine in the same nanoparticle the recognition element ( $S$ sequence) and the amperometric transduction element (OsPA) by layer-bylayer self-assembling. AuNPs were exposed to different concentrations of $S$ representing different $S$ :AuNP molar ratios. In Table 1, the different tested combinations are presented. The nanoparticles were incubated in the presence of different concentrations of $S$ for 1 hour. They were centrifuged at $15000 \mathrm{~g}$ (Scheme 2) and resuspended in 10 mM HEPES buffer, $\mathrm{pH} 7.4$, remaining stable in solution due to the negative charges provided by the oligonucleotide phosphate moieties. The generated combinations (AuNP/Sxx, Table 1) were exposed to the same concentration of OsPA, incubated for 1 hour, centrifuged and resuspended in HEPES buffer. Stable suspensions are obtained in all cases in agreement with previous results. ${ }^{[34-37]}$ 


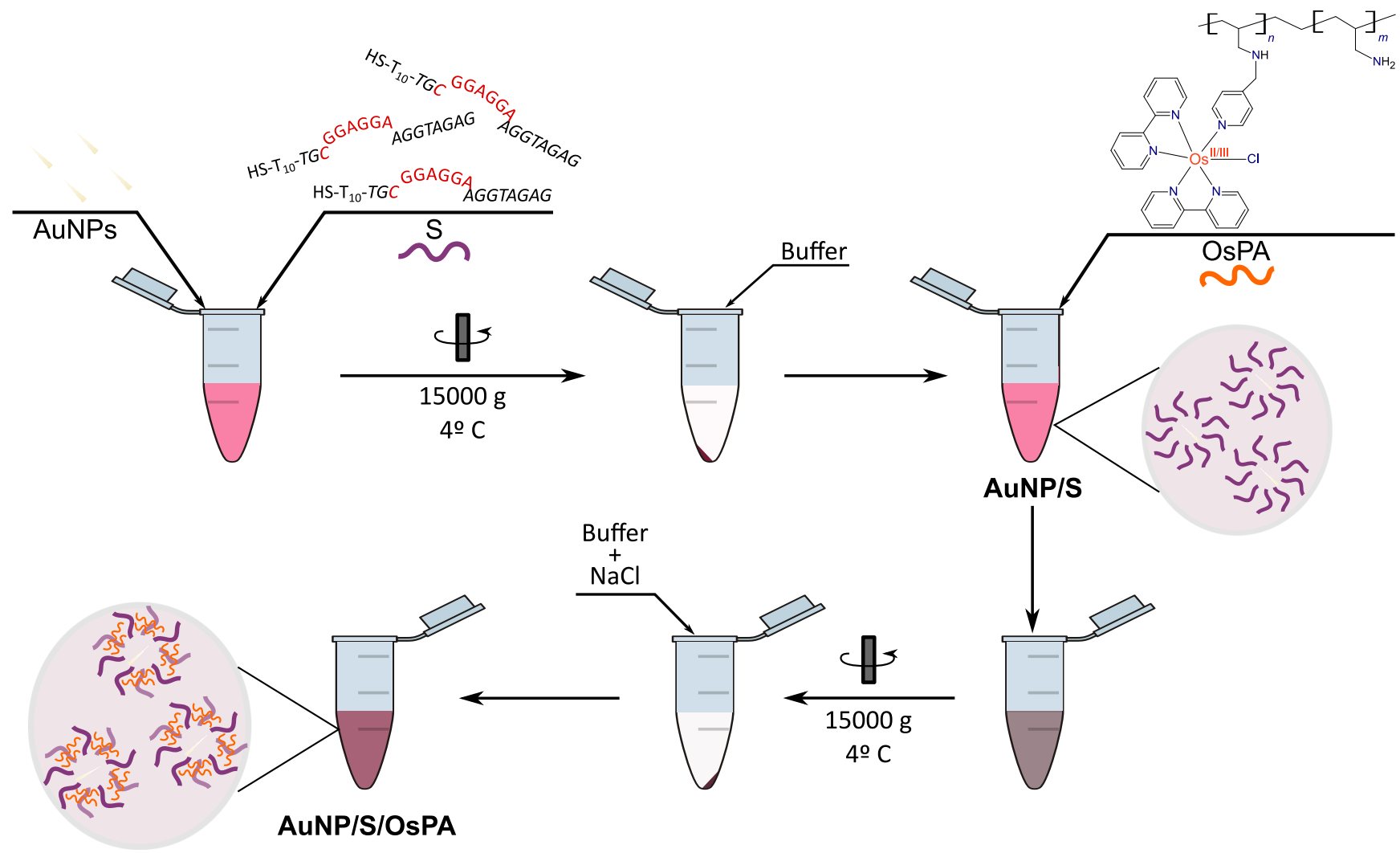

Scheme 2. AuNP modification steps, first with $S$ and then with OsPA.

Table 1. Modification of AuNP with different S concentrations

\begin{tabular}{lcccc}
\hline & AuNP/S10 & AuNP/S05 & AuNP/S02 & AuNP/S01 \\
\hline $12.8 \mathrm{nM}$ AuNP / $\mu \mathrm{L}$ & 50 & 50 & 50 & 50 \\
\hline $10 \mu \mathrm{M} \mathrm{S} / \mu \mathrm{L}$ & 10 & 5 & 2 & 1 \\
\hline $10 \mathrm{mM} \mathrm{HEPES/} \mathrm{LL}$ & 40 & 45 & 48 & 49 \\
\hline final [S]/nM & 1000 & 500 & 200 & 100 \\
\hline S:AuNP [a] & 156 & 78 & 31 & 16
\end{tabular}

[a] Ratio between final concentrations of $S$ and AuNP. For AuNP, the final concentration is $6.4 \mathrm{nM}$ in all cases.

The morphology and the size of the AuNPs were determined by TEM and UV-Vis spectroscopy (Figure 1), from these images, we can observe that AuNPs have $21 \pm 3 \mathrm{~nm}$ diameter $(n=22)$. In Figure 1A, typical aptamer modified AuNPs can be observed, while Figure $1 \mathrm{~B}$ shows the same nanoparticles after modification with the redox polyelectrolyte. The presence of the osmium atoms is evidenced by a different contrast surrounding the AuNP. The UV-Vis spectra obtained before and after functionalization with $S$ present a $2 \mathrm{~nm}$ shift in the maximum wavelength absorbance; while after OsPA functionalization, this shift increases in $4 \mathrm{~nm}$, in agreement with the increase in the AuNPs diameter ${ }^{[6,22]}$ (Figure 1C). Also, from the UV-Vis analysis of the initial solution and the supernatant recovered after AuNP/S/OsPA centrifugation, it was possible to determine the number of osmium probes assembled in each nanoparticle yielding a AuNPs:Os molar ratio of 1:3.1 $\times 10^{4}$.

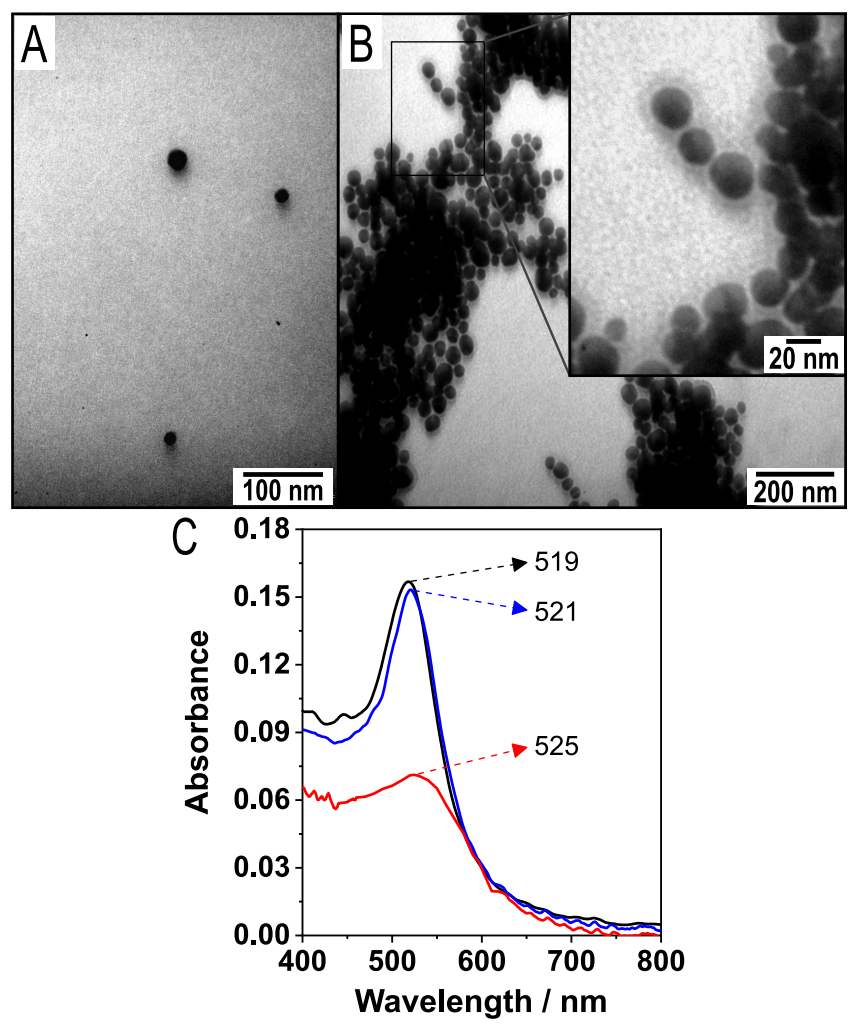

Figure 1. AuNPs characterization. TEM image of $A) A u N P / S$ and $B$ ) AuNP/S/OsPA. C) UV-Vis spectra for AuNP (black), AuNP/S (blue) and AuNP/S/OsPA (red). The numbers indicate the maximum wavelength absorbance in each case. 
The modified nanoparticles (AuNP/S/OsPA) were left to dry onto an electrode surface and rinsed with water. Then, the adsorbed NPs were tested to determine the presence of the osmium probes in the nanoparticle (Figure 2). It can be observed that the amperometric response increases as the amount of $S$ used in the modification of the AuNP increases. This behavior indicates that the adsorption of OsPA is maximized on the nanoparticle surface when the highest $S$ concentration is used. Then, the following experiments have been carried with AuNP/S10/OsPA

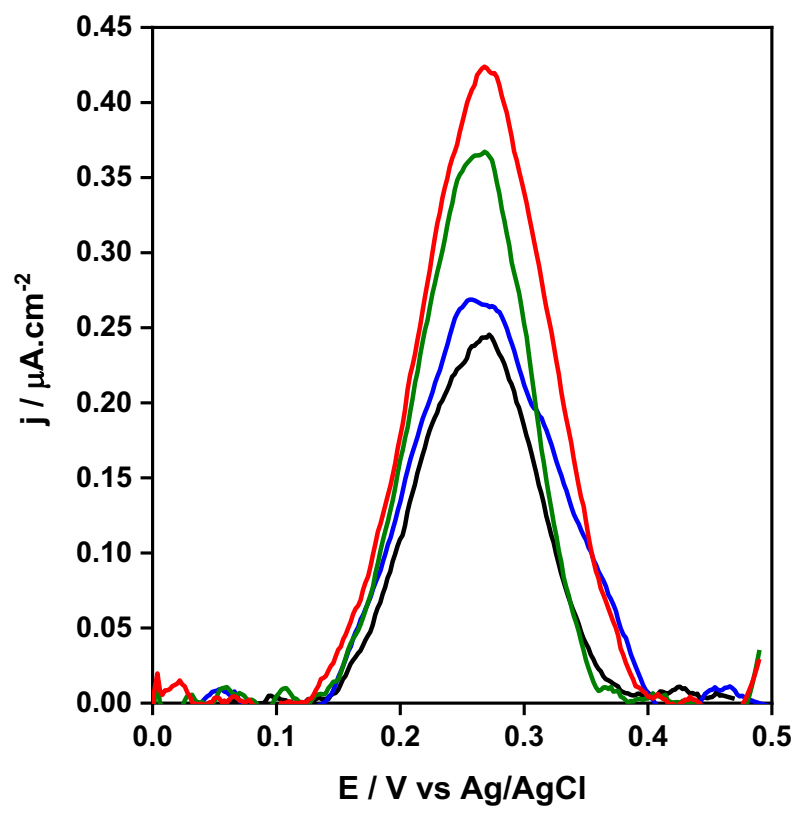

Figure 2. Square wave voltammograms for the different AuNP/Sxx/OsPA: AuNP/S10/OsPA (red), AuNP/S05/OsPA (green), AuNP/S02/OsPA (blue), AuNP/S01/OsPA (black).

\subsection{Surface modification}

Gold electrodes were modified with the thiolated capture aptamer (C) to form a self-assembled monolayer (SAM) (Scheme 1B). Thiolated DNA probes bind to gold through the specific Au-S interaction; however, nonspecific adsorption of DNA occurs on gold due to its interaction with the nitrogen atoms present in each nucleotide. The formation of mixed SAMs with an inert mercapto-derivative improves the aptamer's orientation toward the solution and reduces the nonspecific binding. ${ }^{[38]}$ Among the most used thiolated species, we can find mercaptohexanol ${ }^{[39,40]}$ and polyethyleneglycol; ${ }^{[6,41]}$ the last one has shown a higher resistance to the nonspecific adsorption of DNA and proteins. The gold electrode modification with $\mathrm{C}$ (AuE/C) was complemented with 5000 Da mercaptopolyethyleneglycol (HS-PEG) at two different concentrations, 10 and $150 \mu \mathrm{M}$. The anti-fouling properties of both surfaces were tested, exposing them to AuNP/S10/OsPA for 4 hours. Even though in previous works, the use of $10 \mu \mathrm{M}$ was enough to guarantee a low nonspecific binding, in our assays, a higher concentration of HS-PEG was needed to guarantee good coverage of the surface. In this condition, 150 $\mu \mathrm{M}$, a drastic reduction of nonspecific adsorption was observed (Figure S2, Supporting Information).

\subsection{Assay performance}

Once we obtained the most appropriate electrode surface and modified AuNPs, we optimized the incubation time in adenosine presence. In previous works, using split aptamers and electrochemical detection, short incubation times were used (few minutes) ${ }^{[8,23]}$ however, the detected concentrations ranged from micro- to millimolar. As the analyte and the aptamer concentrations are relevant in the kinetics of the recognition process, we decided to set longer incubation time to achieve the detection at nanomolar levels. We found a signal saturation at $10 \mathrm{nM}$ concentration if we incubate the sample for 4 hours. Therefore, to balance a useful dynamic range with a competitive limit of detection, we reduced the incubation time to two hours, this time is similar to the one used by Wang et al. for low adenosine concentrations. ${ }^{[42]}$ Figure 3 shows the calibration curve obtained for samples ranging from 5 to $75 \mathrm{nM}$ of adenosine per duplicate, with a sensitivity of $82 \mathrm{nA} \mathrm{nM}{ }^{-1} \mathrm{~cm}^{-2}$. Each determination was carried out in a freshly prepared modified electrode and subtracting an average background current of two electrodes produced in the same batch. It can be observed that the dynamic range covers all the tested concentrations, and the calculated limit of detection is $3.1 \mathrm{nM}$ of adenosine (see Supporting Information). Also, good reproducibility of the construction method is achieved since the calibration curve involves 10 independent electrodes (10 samples at 5 different concentrations, each measured in a new disposable electrode). From these series of measurements, only one result was discarded as an outlier.

The major challenges to the assay selective are the presence of nucleobases and their derivatives. These molecules generally generate a small signal, around $2-10 \%$ of the adenosine signal, but always remarkably similar among them..$^{[5,43-46]}$ This fact can be structurally understood considering the NMR solved aptamer structure. Adenosine is a nucleoside consisting of adenine, a nucleobase derivative of purine, attached to a ribose. Lin and Pate ${ }^{[24]}$ established that the aptamer's two recognition domains can locate two adenosine molecules through their adenine bases inside the loop structure. On the other hand, Huizenga and Szostak ${ }^{[20]}$ have established that any modification on the adenine structure difficult the aptamer recognition. In this sense, the guanine base, another purine derivative, and its nucleosides are the most similar molecules to be used as a negative control. In this work, 2'-deoxyguanosine was used to test the selective; Figure 4 shows the results obtained for this molecule against a similar adenosine concentration. The difference between the current obtained in the presence of $75 \mathrm{nM} 2$-deoxyguanosine is lower than the current obtained for $5 \mathrm{nM}$ adenosine. This response is similar to those observed in previous assays,,$[5,43-46]$ indicating that some random interactions between the aptamer and these nucleosides exist, a plausible behavior since all of them are molecules rich in amino and hydroxyl groups that form hydrogen bonds. 


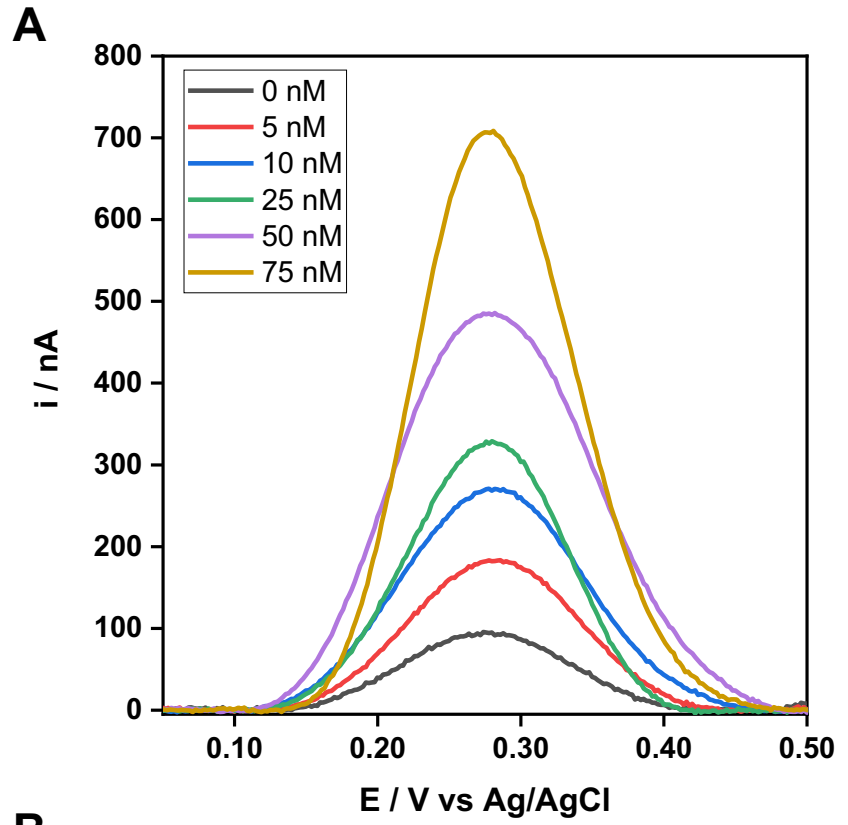

B

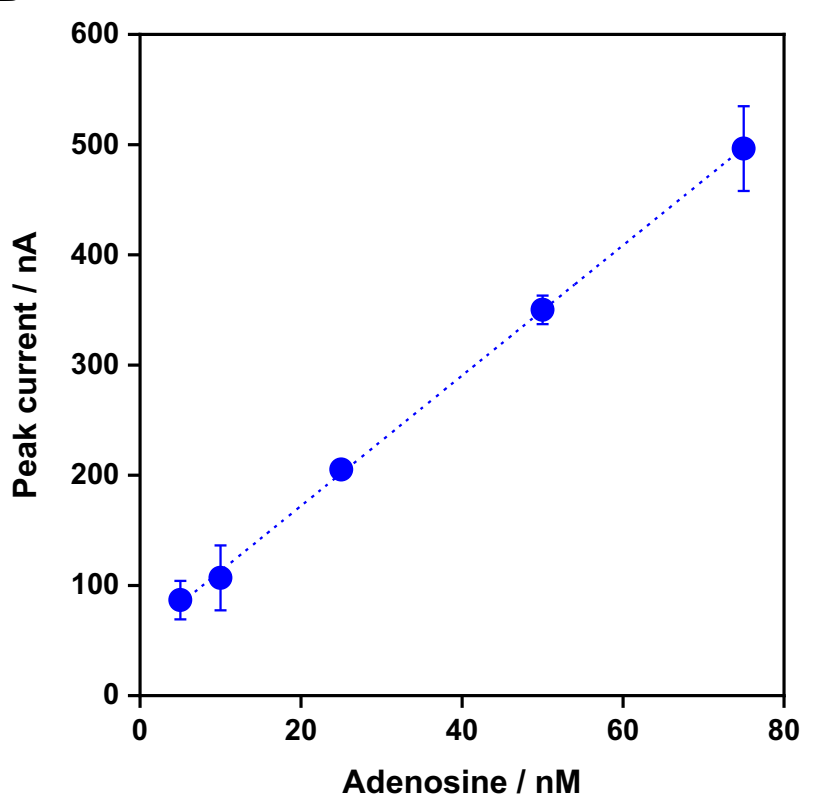

Figure 3. (A)-Square wave voltammograms at different concentrations and (B) Amperometric response as a function of the adenosine concentration. The dotted line corresponds to the linear fitting.

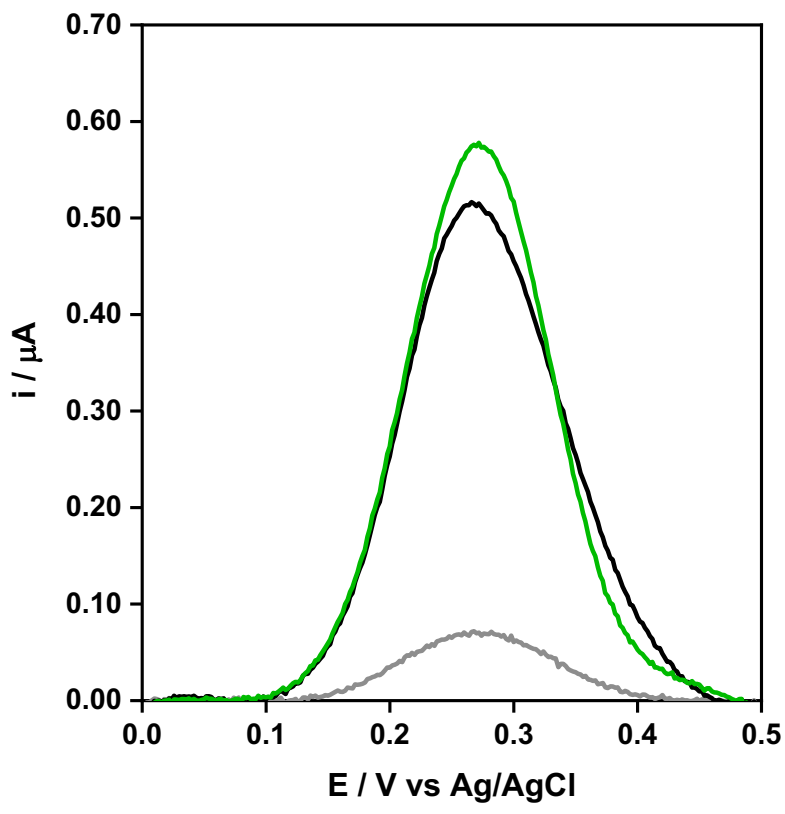

Figure 4. Square wave voltammograms for $75 \mathrm{nM}$ adenosine (black), $75 \mathrm{nM}$ 2'-deoxyguanosine (gray) and $75 \mathrm{nM}$ adenosine plus $75 \mathrm{nM} 2$ 'deoxyguanosine (green)

One exciting feature of the results presented here is the assay's ability to detect a concentration of adenosine as low as $5 \mathrm{nM}$, considering the binding constant of the original aptamer (6 $\mu \mathrm{M}){ }^{[8]}$ This behavior can be explained if we picture each AuNP as a molecule with more than one site to bind the complementary DNA fragments present on the electrode surface. Bearing in mind the size and composition of one nanoparticle, and the area that covers when is bound to the electrode surface, we can check if this represents a reasonable model. We carried out quartz crystal microbalance experiments to evaluate the amount of $C$ fragment adsorbed on gold yielding a surface coverage of $7.7 \mathrm{pmol} \mathrm{cm} \mathrm{cm}^{-2}$. On the other hand, considering the average AuNPs diameter $(21 \mathrm{~nm})$, the projected area of the whole particle onto the surface is $7.7 \times 10^{-12} \mathrm{~cm}^{-2}$. If we consider that the $\mathrm{C}$ sequences are homogeneously distributed on the gold surface, there are around $16 \mathrm{C}$ molecules below the projected area to interact with a single AuNP (outlined in Scheme 3). The number of DNA strands per nanoparticle can be estimated using the results obtained by Mirkin's group: ${ }^{[47]}$ for AuNPs with a 21 $\mathrm{nm}$ diameter, an average of 100 strands per nanoparticle can be considered. Given this number, more than one strand can be found at the bottom part of the AuNP. Therefore, we can argue that the modified nanoparticle is working as a multisite binding nanostructure improving the formation constant of the adenosine aptamer complex. Regarding the signal generation, the presence of more than 30000 osmium probes per each nanoparticle generates a multiplicative effect in the response, improving its sensitivity. 


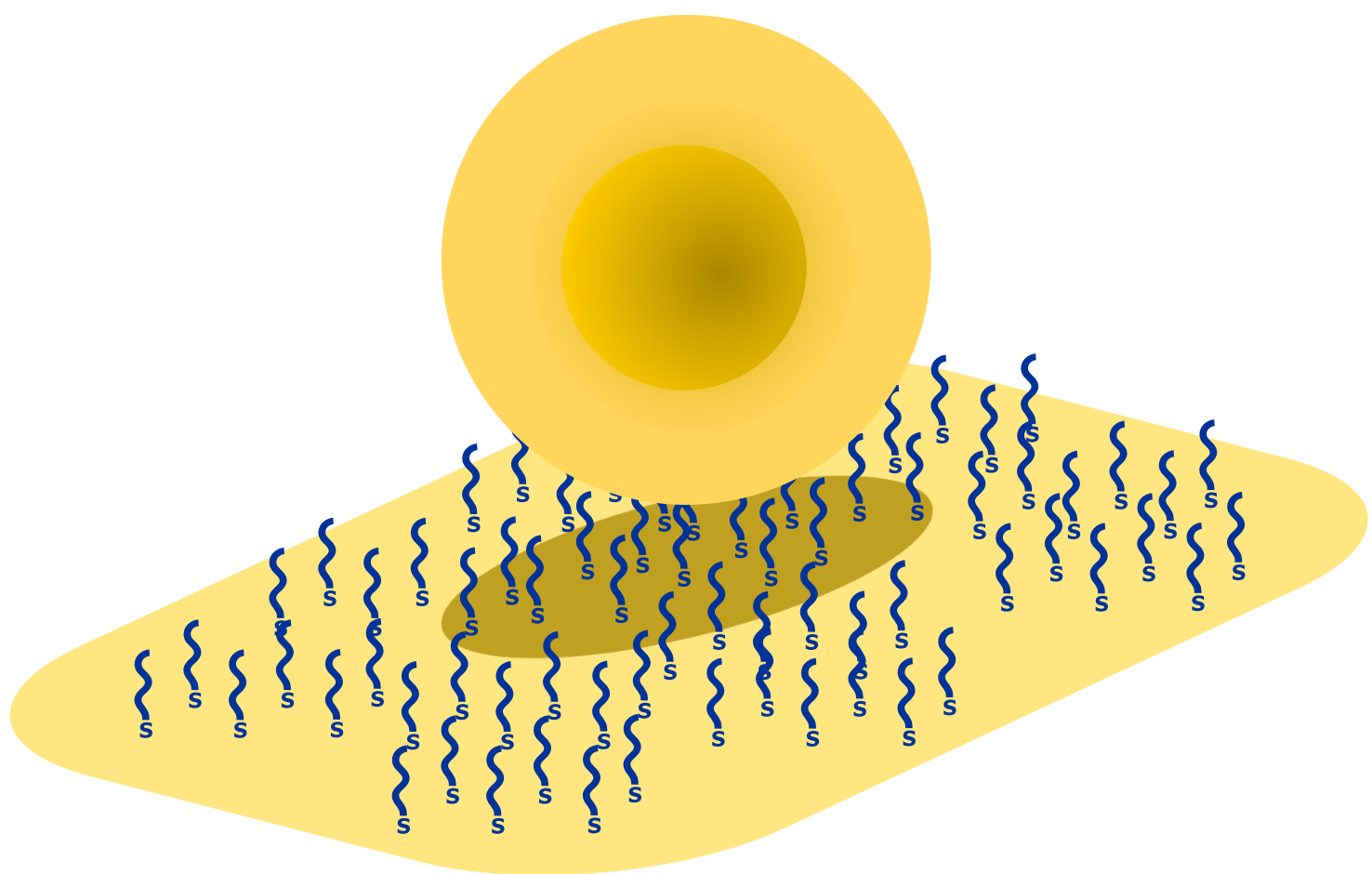

Scheme 3. Possible contact points between AuNP and the electrode surface.

Table 2. Analytical performances of recently published methods for adenosine detection

\begin{tabular}{|c|c|c|c|c|}
\hline Method & $\begin{array}{l}\text { Dynamic } \\
\text { range /nM }\end{array}$ & Response & $\mathrm{LOD} / \mathrm{nM}$ & ref \\
\hline Colorimetry & $5-1000$ & Logarithmic & 0.13 & [48] \\
\hline Fluorometry & Up to 320 & Linear & 2.4 & [49] \\
\hline Electrochemical & $0.1-10^{6}$ & Logarithmic & 0.1 & [43] \\
\hline Electrochemical & $5-200$ & Linear & 5 & [44] \\
\hline Electrochemical & $10-1000$ & Logarithmic & 3 & [45] \\
\hline Electrochemical & $10-40$ & Linear & 2.5 & [46] \\
\hline Fluorometry & $\begin{array}{l}\text { Up to } \\
6000\end{array}$ & Linear & 130 & [50] \\
\hline Photoelectrochemical & $0.01-1000$ & Logarithmic & $3.3 \times 10^{-3}$ & [51] \\
\hline Electrochemical & $5-75$ & Linear & 3.1 & $\begin{array}{l}\text { This } \\
\text { work }\end{array}$ \\
\hline
\end{tabular}

We can consider that each nanoparticle works as a multidentate ligand improving its binding to the surface, achieving a state-ofthe-art adenosine quantification with a limit of detection (LOD) of $3.1 \mathrm{nM}$, as it can be observed in Table 2, where our system is compared to other works published in the last decade. It can be observed in the table that those works presenting an expanded dynamic range have the limitation to scale in a logarithmic way, and the concentration can be determined in a semiquantitative way (orders of magnitude). In particular, the work informing a LOD of $0.1 \mathrm{nM}$ is the result corresponding to the lowest concentration determined, which in turn presents a significant misfit with the determined regression curve (see Figure 4 in the cited reference). ${ }^{[43]}$ It is worth mentioning that in all the cases reviewed for this work the old definition for LOD of $S / N=3$ is used, yielding a more favorable result, while in our system the statistical analysis was carried out according to current IUPAC guidelines (see Supporting Information). ${ }^{[52]}$

\section{Conclusion}

This work describes an electrochemical based sandwich-type assay. AuNPs are modified with a split aptamer and a redox polyelectrolyte. The first DNA fragment is used as a recognition element, while the other for the electrochemical signal generation. The split-aptamer used here can detect a small molecule, represented by adenosine; besides, the increasing development of oligonucleotide engineering allows replication of this strategy to other small molecules of toxicological or metabolic relevance. ${ }^{[10-16]}$ The assay achieves state-of-the-art figures of merit as it can be observed in Table 2.

In our assay, the redox polyelectrolyte surrounded the nanoparticle provides a label that immediately can generate a signal, in contrast to previous electrochemical sandwich immunoassay involving metallonanoparticles, where the signal is generated after several steps involving first a chemical dissolution step, with the introduction of new reagents, followed by an electrochemical step to complete the determination. ${ }^{[17-19,23]}$ Therefore, the combination of two dynamics areas of materials science (nanotechnology and soft matter) can improve the detection limit of a recognition assay by generating the equivalent to a macromolecule constituted by a scaffold with multiple binding sites and multiple signal-generating moieties. This concept is represented here by the AuNP (scaffold) 
containing several aptamers (multiple binding sites) and the redox moieties (signal generation).

These features also lead to other advantages. For example, the analysis can be performed using a low volume of liquid $(25 \mu \mathrm{L})$; eventually, it can be carried out in turbid media, and it involves low-cost instrumentation, represented here by a disposable three-electrode system (Figure S3), that it can be immediately adopted to field-portable devices. In particular, the materials involved in this work are screen-printed electrodes, produced by a well-established technology used in the fabrication of glucose strips $^{[53]}$ and gold nanoparticles, widely used in chromatographic immunoassay. ${ }^{[54]}$ The results presented here regarding linearity and limit of detection, where all the samples were tested on new freshly modified electrodes indicate that the system is reproducible and, therefore, scalable, representing a promising tool in bioassays that can be easily adopted to other analytes, providing a sandwich type recognition system.

\section{Supporting information Summary}

Experimental section and statistical analysis are presented in this part.

\section{Acknowledgements}

The authors wish to acknowledge the support of the NanoAndes IRN program (funded by the CNRS) and the ARCANE Labex (CBH-EUR-GS/ANR-17-EURE-0003),UBACYT 20020170100341BA, and BID PICT 2015-0801.

\section{Conflict of Interest}

The authors declare no conflict of interest.

Keywords: aptamers $\bullet$ biosensors $\cdot$ gold nanoparticle $•$ metabolite $\cdot$ multiple binding site

[1] L. Habartová, K. Hrubešová, K. Syslová, J. Vondroušová, Z. Fišar, R. Jirák, J. Raboch, V. Setnička, Clin. Biochem. 2019, 72, 58-63.

[2] J. R. Ussher, S. Elmariah, R. E. Gerszten, J. R. B. Dyck, J. Am. Coll. Cardiol. 2016, 68, 2850-2870.

[3] R. S. Kelly, M. G. V. Heiden, E. Giovannucci, L. A. Mucci, Cancer Epidemiol. Biomarkers Prev. 2016, 25, 887-906.

[4] T. A. Feagin, N. Maganzini, H. T. Soh, ACS Sensors 2018, 3, $1611-$ 1615.

[5] A. S. Peinetti, H. Ceretti, M. Mizrahi, G. A. González, S. A. Ramírez, F. G. Requejo, J. M. Montserrat, F. Battaglini, Nanoscale 2015, 7, 7763-7769.

[6] F. Melaine, C. Coilhac, Y. Roupioz, A. Buhot, Nanoscale 2016, 8, 16947-16954.

[7] F. Li, J. Zhang, X. Cao, L. Wang, D. Li, S. Song, B. Ye, C. Fan, Analyst 2009, 134, 1355-1360.

[8] X. Zuo, Y. Xiao, K. W. Plaxco, J. Am. Chem. Soc. 2009, 131, 69446945.

[9] R. Freeman, E. Sharon, R. Tel-Vered, I. Willner, J. Am. Chem. Soc. 2009, 131, 5028-5029.
M. Debiais, A. Lelievre, M. Smietana, S. Müller, Nucleic Acids Res. 2020, 48, 3400-3422.

[11] T. Guo, C. Wu, A. Offenhäusser, D. Mayer, Phys. Status Solidi A 2020, 1900924.

[12] Y. Luo, H. Yu, O. Alkhamis, Y. Liu, X. Lou, B. Yu, Y. Xiao, Anal. Chem. 2019, 91, 7199-7207.

[13] M. A. Nameghi, N. M. Danesh, M. Ramezani, M. Alibolandi, K. Abnous, S. M. Taghdisi, Anal. Chim. Acta 2019, 1065, 107-112.

[14] M. A. D. Neves, S. Slavkovic, O. Reinstein, A. A. Shoara, P. E. Johnson, RSC Adv. 2019, 9, 1690-1695.

[15] A. K. Sharma, J. M. Heemstra, J. Am. Chem. Soc. 2011, 133 12426-12429.

[16] Z. Zhang, X. Zhao, J. Liu, J. Yin, X. Cao, Sens. Actuators, B 2020 311,127920

[17] M. Dequaire, C. Degrand, B. Limoges, Anal. Chem. 2000, 72, 55215528.

[18] D. Kalyoncu, Y. Tepeli, U. C. Kirgöz, A. Buyraç, Ü. Anik, Electroanalysis 2017, 29, 2832-2838.

[19] N. E. Pollok, C. Rabin, C. T. Walgama, L. Smith, I. Richards, R. M. Crooks, ACS Sensors 2020, 5, 853-860.

[20] D. E. Huizenga, J. W. Szostak, Biochemistry 1995, 34, 656-665.

[21] H. Zhang, Y. Liu, K. Zhang, J. Ji, J. Liu, B. Liu, Anal. Chem. 2018 90, 9315-9321.

[22] F. Melaine, Y. Roupioz, A. Buhot, Microarrays 2015, 4, 41-52.

[23] L. Kashefi-Kheyrabadi, M. A. Mehrgardi, Biosens. Bioelectron. 2012, 37, 94-98.

[24] C. H. Lin, D. J. Patei, Chem. Biol. 1997, 4, 817-832.

[25] Y. Roupioz, J. Chem. Educ. 2019, 96, 1002-1007.

[26] C. Lu, C. Saint-Pierre, D. Gasparutto, Y. Roupioz, E. Peyrin, A. Buhot, Langmuir 2017, 33, 12785-12792.

[27] J. J. Richardson, J. Cui, M. Björnmalm, J. A. Braunger, H. Ejima, F. Caruso, Chem. Rev. 2016, 116, 14828-14867.

[28] J. Borges, J. F. Mano, Chem. Rev. 2014, 114, 8883-8942.

[29] M. Tagliazucchi, E. J. Calvo, ChemPhysChem 2010, 11, 2957-2968.

[30] E. Tjipto, J. F. Quinn, F. Caruso, J. Polym. Sci., Part A Polym. Chem. 2007, 45, 4341-4351.

[31] F. Caruso, D. N. Furlong, K. Ariga, I. Ichinose, T. Kunitake, Langmuir 1998, 14, 4559-4565.

[32] M. L. Cortez, A. L. Cukierman, F. Battaglini, Electrochem. commun. 2009, 11, 990-993.

[33] D. Wang, Q. Liu, D. Wu, B. He, J. Li, C. Mao, G. Wang, H. Qian, ACS Appl. Mater. Interfaces 2018, 10, 15504-15516.

[34] D. Brondani, B. de Souza, B. S. Souza, A. Neves, I. C. Vieira, Biosens. Bioelectron. 2013, 42, 242-247.

[35] V. Cebrián, F. Martín-Saavedra, C. Yagüe, M. Arruebo, J. Santamaría, N. Vilaboa, Acta Biomater. 2011, 7, 3645-3655.

[36] P. Scodeller, V. Flexer, R. Szamocki, E. J. Calvo, N. Tognalli, H. Troiani, A. Fainstein, J. Am. Chem. Soc. 2008, 130, 12690-12697.

[37] M. L. Cortez, W. Marmisolle, D. Pallarola, L. I. Pietrasanta, D. H. Murgida, M. Ceolín, O. Azzaroni, F. Battaglini, Chem. - A Eur. J. 2014, 20, 13366-13374.

[38] D. Li, S. Song, C. Fan, Acc. Chem. Res. 2010, 43, 631-641.

[39] T. M. Herne, M. J. Tarlov, J. Am. Chem. Soc. 1997, 119, 8916-8920.

[40] Y. Wan, J. Zhang, G. Liu, D. Pan, L. Wang, S. Song, C. Fan, Biosens. Bioelectron. 2009, 24, 1209-1212.

[41] J. Zhang, R. Lao, S. Song, Z. Yan, C. Fan, Anal. Chem. 2008, 80, 9029-9033. 
G. Wang, Q. Xu, L. Liu, X. Su, J. Lin, G. Xu, X. Luo, ACS Appl. Mater. Interfaces 2017, 9, 31153-31160.

[43] D. Zhang, J. Ma, X. Meng, Z. Xu, J. Zhang, Y. Fang, Y. Guo, Anal. Chim. Acta 2019, 1076, 55-63.

[44] C. Sun, X. Liu, K. Feng, J. Jiang, G. Shen, R. Yu, Anal. Chim. Acta 2010, 669, 87-93.

[45] F. Yan, F. Wang, Z. Chen, Sens. Actuators, B 2011, 160, 13801385.

[46] D. Wu, X. Ren, L. Hu, D. Fan, Y. Zheng, Q. Wei, Biosens. Bioelectron. 2015, 74, 391-397.

[47] L. M. Demers, C. A. Mirkin, R. C. Mucic, R. A. Reynolds, R. L. Letsinger, R. Elghanian, G. Viswanadham, Anal. Chem. 2000, 72, 5535-5541.

[48] C. Kong, L. Gao, Z. Chen, Microchim. Acta 2018, 185, 488.

[49] J. You, Z. You, X. Xu, J. Ji, T. Lu, Y. Xia, L. Wang, L. Zhang, S. Du, Microchim. Acta 2019, 186, 43.

[50] W. Li, J. Sun, H. Wang, L. Wang, W. Jiang, Sens. Actuators, B 2018 260, 581-586.

[51] Y. Gao, H. Qi, M. Shang, J. Zhang, J. Yan, W. Song, Biosens. Bioelectron. 2019, 146, 111741.

[52] A. C. Olivieri, Anal. Chim. Acta 2015, 868, 10-22.

[53] A. Heller, B. Feldman, Chem. Rev. 2008, 108, 2482-2505.

[54] C. A. Burtis, E. R. Ashwood, D. E. Bruns, Tietz Textbook of Clinical Chemistry and Molecular Diagnostics., Elsevier Health Sciences, St. Louis, Missouri, USA, 2012. 


\section{Entry for the Table of Contents}

Insert graphic for Table of Contents here. ((Please ensure your graphic is in one of following formats))

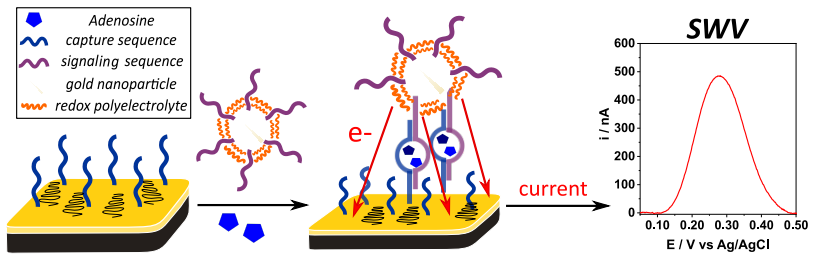

Integrating advantages. Modification of AuNP with a split aptamer and a redox polyelectrolyte produces a sensitive electrochemical sandwich assay for adenosine; the redox polyelectrolyte surrounded the nanoparticle provides a label that immediately can generate a signal using square wave voltammetry on a modified screen-printed electrode. The method's sensitivity opens new alternatives for detecting metabolites through a simple amperometric assay using well-established components of the dignositics industry. 